\title{
Lames de fond historiques et spécificités nationales
}

\section{Anne Van Haecht}

Il est fascinant de constater combien les acteurs du changement scolaire se sont servis des mêmes arguments en France, dans le Canton de Genève et en Belgique pour défendre le projet de «l'école unique» et combien ils l'ont fait tous à partir de l'après-guerre 1914-1918. Et pourtant l'adoption de l'obligation scolaire n'y avait guère été le résultat d'un cheminement similaire. C'est à Genève que la disposition a été la plus précoce (1872) dans un contexte rendu favorable en raison de la présence de forces politiques suffisamment fortes pour empêcher la suprématie d'une Eglise ou d'un point de vue idéologique conservateur (à la différence évidente du Valais ${ }^{1}$ ). En France, la figure de Jules Ferry ne permet néanmoins pas de faire l'impasse sur l'œuvre préalable de Guizot, aussi conservatrice ait-elle été. En Belgique, par contre, l’instruction obligatoire a été vraiment tardive (loi du 19 mai 1914), ce qui s'explique par les modes de régulation de cet Etat faible dès sa naissance (1830). La Constitution belge (1831) a proclamé la liberté d'enseignement dans un pays qui allait connaître deux guerres scolaires (1878-1884 et 1954-1958), dues à la rivalité - toujours réelle aujourd'hui - opposant les réseaux scolaires dépendant des pouvoirs publics (Etat, provinces, villes et communes) et le réseau libre catholique. Ce sont les libéraux progressistes d'abord (cf. l'action de la Ligue de l'Enseignement créée en 1864), suivis par les socialistes et les démocrates chrétiens qui ont fini par l'obtenir à l'usure, selon une structure attribuant ici aussi à l'école primaire l'allure d'un ordre séparé par rapport à l'ordre secondaire, grâce à l'existence d'un "quatrième degré» (de 12 à 14 ans) pour les jeunes destinés au travail précoce.

C'est donc dès l'après Première Guerre mondiale que dans ces trois contextes nationaux une même revendication va émerger, à partir des milieux laïques et progressistes, en vue de la réalisation d'une "école unique». En Belgique, elle sera relayée dès les années ' 20 dans de nombreux textes et déclarations stigmatisant l'impasse que représente le quatrième degré primaire et condamnant l'inexistence d'un "principe de continuité» entre l'enseignement professionnel et l'enseignement moyen. Un des idéaux socialistes typiques de l'époque était encore de permettre à un jeune de milieu pauvre, capable et forcément méritant, de passer 
d'une école professionnelle à une école d'ingénieur technicien pour accéder ensuite à une Faculté de sciences appliquées. L’idée centrale des partisans de l'école unique était donc de créer des passerelles autorisant tout individu à se réorienter pour mieux continuer des études et de pratiquer une sélection par l'intelligence seule. Dans notre pays où le projet de Jean Zay (1937) et le plan Langevin-Wallon (1947) ont connu une large audience dans les cercles pédagogiques laïques, ce sont des ministres socialistes qui, des années 1940 aux années 1960, poseront les jalons de nouvelles structures pour l'enseignement obligatoire, officialisant enfin l'enseignement secondaire "rénové» (un cycle d'observation, suivi d'un cycle d'orientation, suivi d'un cycle de détermination) en 1971. C'est donc la gauche (socialistes et démocrates chrétiens) qui, en Belgique, a fait aboutir le projet. Mais il faut reconnaître que ce fut, in extremis, sans grande difficulté. En effet, la réforme était soutenue par les organisations internationales (le Conseil de l'Europe en tête) qui prônaient l'assouplissement des structures scolaires avec un tronc commun minimal, le recours à des méthodes pédagogiques individualisantes, la participation et la gestion associative au sein des écoles, la démocratisation de l'accès à l'enseignement supérieur, etc. Les libéraux et les catholiques conservateurs s'y sont ralliés d'autant plus facilement que l'existence d'un enseignement secondaire «traditionnel» était préservée à côté de la création du «rénové» (cf. Van Haecht, 1985). De plus, le système politique belge, "pilarisé»2 avec ses différents réseaux scolaires, laissait toute liberté à ceux-ci pour se rénover ou non et pour décider de leurs méthodes pédagogiques propres.

Des années ' 50 au début des années '70, à la faveur d'un contexte économique de croissance, des thématiques similaires, porteuses des mêmes injonctions, ont donc fini par l'emporter, au moins de manière rhétorique, dans les trois politiques scolaires spécifiques concernées ici alors que les rapports de force en présence différaient. Mais en deçà d'un esprit de réforme qui paraissait général, les institutions modifiées conservaient leur spécificité. Reste que toutes paraissent à postériori avoir été conçues pour autoriser le maintien d'une différenciation plus ou moins rapide des élèves. Ainsi en va-t-il certainement pour la Belgique et la France. Dans le premier cas, on a prévu dès la première année du cycle d'observation du "rénové» l'ouverture d'une première année $B$ à côté de la première $\mathrm{A}$ afin d'y accueillir les élèves en difficulté. Conçue pour permettre une remédiation et éventuellement une réorientation vers l'enseignement général normal, cette première année $B$ est devenue très rapidement l'antichambre d'un enseignement professionnel qui, dans ce pays, constitue une véritable voie de relégation. Dans la suite du cursus, la suppression des anciennes «sections» d'humanités anciennes et modernes (latin-grec, latin-mathématiques, scientifiques, etc.) s'est doublée de la création de nombreuses filières optionnelles, évidemment hiérarchisées les unes par rapport aux autres. L'injonction «Travaillez, sinon vous serez orienté», lancée, d'après Antoine Prost, aux élèves du collège unique français, va dans le même sens, ainsi que toute une série de pratiques distinctives dont ont pu s’inspirer les parents culturellement éclairés. Ici et là, la résistance 
des inégalités sociales s'est manifestée dès la concrétisation (tardive) des mesures s'inscrivant dans la foulée de «l'école unique».

La formule selon laquelle «en matière d'égalité d'accès à l'instruction, c'est l'économique qui dope le politique», appliquée par Charles Magnin (cf. supra p. 35) à la démocratisation du système scolaire genevois, se révèle tout aussi vraie dans les autres pays. Mais en période de vaches maigres, c'est aussi l'économique qui bloque le politique. Antoine Prost a bien montré combien la proposition de Chevènement en 1985 de conduire au niveau bac 80\% de la classe d'âge en l'an 2000, suivie d'une croissance sensible des lycées, s'est trouvée un peu plus tard en contradiction avec des contraintes économiques de plus en plus manifestes. En Belgique, depuis que chaque Communauté linguistique est responsable financièrement de son système scolaire (1988), la Communauté Française a opéré de nombreuses mesures de rationalisation budgétaire et un esprit critique pourrait se demander, entre autres, si certaines interdictions de faire redoubler un élève à l'intérieur d'un cycle d'études dans l'enseignement obligatoire ne constituent pas davantage des mesures d'économies déguisées que des décisions à haute teneur pédagogique ...

Dans nombre de pays, comme le dit Antoine Prost pour la France, le souffle égalitaire animant les politiques scolaires de l'après-guerre est retombé et il en reste sans doute quelque désenchantement. Mais est-il vrai pour autant, comme il le note à la fin de son article (cf. supra, p. 90), que «le passage de la démocratisation à la lutte contre l'échec scolaire annonce peut-être un recentrage modeste de l'école sur des objectifs purement scolaires»? Tout d'abord la lutte contre l'échec scolaire ne parait guère être devenue un défi relevé avec succès, sauf à tenter d'éluder l'échec sous forme de "réorientations» discréditantes en cascade. Mais ensuite et plus largement l'école ici convoquée n'existe vraisemblablement plus, sinon à se replier sur la maternelle et le primaire, et encore. Depuis quelques vingt ans déjà, l'institution scolaire a perdu son monopole dans un champ qui était le sien et le sien seul, et fait dorénavant partie d'un monde plus large, celui d'un quasi-marché des formations où elle figure parmi un ensemble d'agences dispensatrices d'attestations de "compétences» - formulation au demeurant politiquement discutable. Avec des slogans aussi invasifs sur ce marché que l'impératif de «l'éducation tout au long de la vie», servant le cas échéant de caution cynique à une segmentation inégalitaire des filières de scolarisation et de formation professionnelle, nous voilà loin d'une possibilité de retour vers des objectifs bien rodés. C'est un chantier radicalement différent qui s'offre désormais à la réflexion sociologique, dans un environnement saisi par l'appel néolibéral à la globalisation, ou moins brutalement dit par l'obligation d'harmonisation des certifications (cf. Van Haecht, 1998).

Et enfin quelques mots trop brefs sur la démarche socio-historique pour conclure. Antoine Prost se réfere volontiers à Paul Veyne pour définir l'approche historique comme une «mise en intrigue» où, comme dans un scénario, les dates ont la valeur de "points de repère commodes" (Prost, 1997, p. 222) qui dési- 
gnent les moments où le changement s'opère. Si je suis d'accord avec lui sur la «mise en intrigue» nécessaire, je pense cependant que ces dates ne s'imposent pas comme telles au chercheur qui n'aurait qu'à suivre «le cours des choses», mais lui non plus sans nul doute. La problématisation heuristique me semble première et obligée. François Furet l'a bien énoncée sous la forme suivante: «il faut d'abord définir des objets de recherche et examiner ensuite, en tâtonnant, quelle est l'épaisseur de durée pertinente pour leur description et leur analyse» (Furet, 1982, p. 12). Construire l'objet, préciser la temporalité dans laquelle il s'inscrit, voilà qui renvoie à un rapport aux valeurs qui est évidemment celui d'un individu particulier qui fait donc le choix de l'intrigue, une parmi d'autres, dont il tentera de renouer les fils avec un regard socialement situé. Même si, une fois l'objet ainsi défini, la méthode pragmatique, scientifiquement fondée dans l'apport de la preuve telle que l'envisage Charles Magnin, est de rigueur. La temporalité propre à l'histoire de «l'école unique» dans tous ses développements apparaît clairement dans ce dossier. Mais réduire l'échec de ce projet à l'existence d'effets pervers au sens où l'entend Boudon n'est pas suffisant: l'agrégation d'actions individuelles me semble un phénomène social inapte à rendre compte précisément de l'historicité. Aussi je dirais que l'histoire des politiques d'éducation est une partie de la sociologie des rapports de domination mise en perspective diachronique, plutôt qu' «une sociologie des organisations mise en perspective diachronique», fût-elle en même temps, comme le dit Antoine Prost, "une histoire des stratégies plurielles d'acteurs sociaux eux-mêmes pluriels» (1997, p. 232). Et il faut encore ajouter sans grande originalité que l'histoire n'est pas le fait de forces impersonnelles. A partir des individus, de certains individus plus que d'autres, il est possible de saisir du social historiquement situé. Pour la démocratisation des études en Europe occidentale, la figure de Jean Zay se place incontestablement parmi ces personnages à partir desquels «nous pouvons connaître le social en partant de la spécificité irréductible d'une praxis individuelle», pour reprendre la formule de Ferrarotti (1983, p. 51) qui opte pour une anthropologie sociale voyant dans chaque homme «la réappropriation singulière de l'universel social et historique qui l'environne» (1983, p. 51).

\section{Notes}

1 Dans la mesure où c'est la question de l'école unique qui m'a retenue essentiellement, je n'ai guère pu introduire dans la discussion les apports très intéressants du texte de Danièle Périsset Bagnoud qui concerne l'histoire de l'Ecole normale au Valais.

2 La Belgique s'est constituée sur la base d'un pluralisme segmenté, c'est-à-dire sur la base de la coexistence de grandes familles politiques (catholique, libérale et, depuis 1885, socialiste) que l'on désigne comme des "piliers». Chaque pilier a développé un réseau d'organisations (culturelles, socio-économiques, politiques, etc.) qui prennent en charge les individus "de la naissance à la mort». Lécole et les organisations éducatives sont parties prenantes de ce système pilarisé. 


\section{Références bibliographiques}

Ferrarotti, F. (1983). Histoire et histoires de vie. Paris: Librairie des Méridiens.

Furet, F. (1982). L'atelier de l'histoire. Paris: Ed. Flammarion.

Prost, A. (1997). Education, société et politiques. Paris: Ed. du Seuil.

Van Haecht, A. (1985). L'enseignement rénové, de l'origine à l'éclipse. Bruxelles: Editions de l'Université de Bruxelles.

Van Haecht, A. (1998). L'école à l'épreuve de la sociologie, questions à la sociologie de l'éducation. (2ème éd). Bruxelles \& Paris: Ed. De Boeck \& Ed. Universitaires. 
Diskussion 